\title{
Effects of alcohol and practice on choice reaction time
}

\author{
E. A. MAYLOR and P. M. A. RABBITT \\ University of Manchester, Manchester, England
}

\begin{abstract}
Forty subjects participated in a four-choice reaction time experiment in which they received alcohol ( $1 \mathrm{ml} / \mathrm{kg}$ body weight) in one session and no alcohol in another on consecutive days (the order being counterbalanced). Fifty practice trials and then 2,000 experimental trials were given in each session. Subjects were slower, more variable, and less accurate overall with alcohol than without. They were also slower and more variable in the first than in the second session. By combining the data across subjects to produce speed-error tradeoff functions, it appears that practice has little effect whereas alcohol has a substantial effect; responses made within 600 msec of stimulus presentation are more likely to be erroneous with alcohol than without. In addition, the number of consecutive errors was increased by alcohol. The results are interpreted within a model in which it is suggested that the primary effect of alcohol upon performance in speeded tasks is to decrease the rate of accumulation of evidence.
\end{abstract}

Although there have been many investigations into the effects of alcohol on reaction time, few definite conclusions can be drawn from them. For example, Drew, Colquhoun, and Long (1959) pointed out the methodological deficiency in several papers of failing to take account of practice effects. In a review of work published between 1940 and 1960, Carpenter (1962) concluded that the quality and sophistication of most experiments on alcohol and reaction time were very low.

While the majority of recent results indicate that "alcohol causes a slowing of reaction time in choice reactiontime tasks"' (Schneider, Dumais, \& Shiffrin, 1984, p. 15), exceptions include the results of Wilkinson and Colquhoun (1968) and Shillito, King, and Cameron (1974). The former study investigated the effect of alcohol on Leonard's (1959) five-choice serial self-paced reaction time task. This uses five lamps, arranged in a pentagon, each adjacent to one of five associated metal contacts. Subjects were required to touch each contact as soon as possible after the lamp next to it was lit. A correct response switched off the lamp and immediately activated the next signal in a pseudorandom sequence. There was no effect on speed and gaps (the number of responses made in a given time and response times more than $1.5 \mathrm{sec}$, respectively), but more errors were made with alcohol, particularly toward the end of a session. Shillito et al. (1974) also found no effect on reaction time, and surprisingly obtained the lowest error rate, not for the placebo, but for a low dose of alcohol.

This research was supported by the Medical Research Council of Great Britain (Grant G8221479). The authors are grateful to Dorothy Bishop for writing the computer programs and Robert Proctor for helpful comments on an earlier version of the paper. Requests for reprints should be addressed to Elizabeth Maylor at the Age and Cognitive Performance Research Centre, University of Manchester, Oxford Road, Manchester M13 9PL, England.
However, in these and other studies (Huntley, 1972, 1974; Tharp, Rundell, Lester, \& Williams, 1974), speed and error measures have been discussed independently and few have acknowledged the possibility of speed-error tradeoffs (SETOs), that is, the ability to increase speed at the expense of accuracy and vice versa. Thus, variations in response criteria can produce performance changes quite apart from the effects of alcohol. Jennings, Wood, and Lawrence (1976) investigated this by generating speed-error tradeoff functions (SETOFs) for several doses of alcohol. An auditory reaction time task with a deadline procedure was used, in which a response had to be made prior to the onset of a visual signal. The delay of the deadline signal varied between 175 and $375 \mathrm{msec}$ in steps of $50 \mathrm{msec}$ but was constant over a block of trials. From the overall results, it was not possible to separate criterion changes from real changes in efficiency as both mean correct reaction time and proportion correct decreased with increasing amount of alcohol. Thus, SETOFs for each dose were generated in the following way. For each deadline condition, mean reaction time was calculated and an accuracy measure was derived from a transformation of the proportion correct. A linear regression was then carried out producing a slope and intercept for each alcohol condition. There was no effect of alcohol on the intercept, but there was a decrease in slope with increasing alcohol. For all doses (.33 to $1.33 \mathrm{ml}$ of $95 \%$ ethyl alcohol per kilogram of body weight), alcohol impaired performance by decreasing the rate of improvement in accuracy with response time. This could be attributed to impaired perceptual analysis of signals (see Broadbent, 1971; Fisher, 1984; Rabbitt, 1979), so that either response time is increased to maintain a stable error rate or speed is unaffected while more errors are made.

The aim of the present experiment was to produce SETOFs with and without alcohol, using a self-paced 
rather than an externally paced task, allowing the subject to exercise control over timing. Rabbitt (1979) suggested that in order to control performance in a self-paced choice reaction time task, subjects must (1) know when errors are made, (2) be able to vary response speed and control it in order to "track" their fastest, safe reaction time band, and (3) know when they make unnecessarily slow responses. For example, elderly subjects have slower mean reaction times than young subjects mainly as a result of impairments in the second and third aspects of control. Large numbers of trials were presented in the present experiment in an attempt to produce reliable SETOFs and to study possible interactions between alcohol and practice. In addition, from a detailed analysis of responses immediately before and after errors, the effect of alcohol on the three control mechanisms could be investigated.

Designs in which subjects are tested first without alcohol and then with alcohol are economical in that the experiment can be completed in one session, but highly questionable because of order effects that are difficult to assess. One way to control for these is to compare the performance of a no-alcohol group with that of an alcohol group. However, as noted by Huntley (1973), "when the twogroup design is used and performance on the task of interest is highly variable, because of the masking effects of individual differences ... very strong treatment effects are frequently necessary in order to obtain statistically significant differences"' (p. 153). The present study therefore employed a design in which half of the subjects received no alcohol in the first session and alcohol in the second; the order for the other subjects was reversed.

\section{METHOD}

\section{Subjects}

Thirty-six male and 4 female volunteers were recruited through advertisements placed around the University of Manchester. They were mostly undergraduates aged between 18 and 37 years (mean $=22.6$ ), and their weights ranged from 55 to $84 \mathrm{~kg}($ mean $=69.1)$. Each subject participated in two sessions, each $1 \mathrm{~h} 20 \mathrm{~min}$ long, at the same time on consecutive days. The present experiment was the third of three carried out during the session. The subjects were paid $£ 6$ for participating in the study.

\section{Apparatus and Stimuli}

The subjects were given alcohol in the form of vodka (37.5\% alcohol by volume) mixed with concentrated orange juice and water (see Procedure for exact quantities). A Lion Alcolmeter S-D2 (breathalyzer) obtained from Lion Laboratories Ltd., Barry, UK, was used to estimate blood alcohol concentration to the nearest $5 \mathrm{mg}$ alcohol $/ 100 \mathrm{ml}$ blood.

An Apple Ile microcomputer was used to control the experiment and record responses using a millisecond clock by Mountain Hardware. The stimuli appeared in the center of a Hitachi $200-\mathrm{mm}$ monitor (white on gray) and were the characters A, B, C, D, each measuring approximately $2.5 \times 3.5 \mathrm{~mm}$. The response keys were " $\mathrm{Z}$ ", " $\mathrm{X}$ ", ",", and "." of the computer keyboard. (This particular letter-identification task has been used in other studies in our laboratories, for example, in the investigation of the effects of age by Rabbitt \& Goward, 1986.)

\section{Design and Procedure}

The subjects were asked to drink no alcohol on the previous evening and to eat nothing for at least $2 \mathrm{~h}$ before each session. Excessive drinkers and teetotalers were excluded from the study following completion of a personal-details questionnaire that included questions on age, weight, number of cigarettes smoked per day, current medication, serious illnesses, and general drinking habits. In addition, the subjects signed a form agreeing to take part in the experiment and to refrain from driving, cycling, and handling dangerous machinery for $24 \mathrm{~h}$. The subjects also agreed to inform the experimenter of any side effects experienced as a result of the alcohol.

Each session began with the subject's drinking $500 \mathrm{ml}$ as quickly as possible. For the "alcohol"' session, this was made up of $150 \mathrm{ml}$ concentrated orange juice, $100 / 37.5 \mathrm{ml}$ vodka (that is, $1 \mathrm{ml}$ alcohol) per kilogram of body weight, and water. For the other session, $150 \mathrm{ml}$ orange juice was mixed with $350 \mathrm{ml}$ water and a few drops of vodka were floated on the top of the drink. The order of the alcohol and no-alcohol sessions was counterbalanced across subjects. The nonalcoholic drink was consumed in an average of $4.25 \mathrm{~min}(S D=1.26)$; the alcoholic drink was consumed in $6.63 \mathrm{~min}(S D=2.74)$. The subject was given no information concerning the contents of the drink. (Most subjects were probably aware of the fact that one drink contained more alcohol than the other. However, it is unlikely that the main results of interest were in any way influenced by this knowledge.) A breath reading was taken $20 \mathrm{~min}$ after completion of the drink, and the present experiment began $5 \mathrm{~min}$ after that. (Two other unrelated experiments were carried out before the present one and in the same session-see Maylor \& Rabbitt, 1987.) At the end of the session ( $80 \mathrm{~min}$ ), a final breath reading was taken.

The subjects were tested individually. Ten blocks of trials of the choice reaction time task were given in each session. There were 250 trials in Block 1 and 200 trials in each of Blocks 2-10. The first 50 trials in Block 1 were regarded as practice and were not included in the analysis. The subject was shown the four possible stimuli (A, B, C, and D) and the corresponding keys (" $\mathrm{Z}$ ", " $\mathrm{X}$ ", ",,", and ".") to be pressed by the index and middle fingers of the two hands. To begin each block, the subject was required to press the spacebar. This was followed by a short delay $(1.5 \mathrm{sec})$ before the presentation of the first stimulus (A, B, C, or D). The subject was required to press the correct key as quickly as possible. This immediately removed the stimulus from the screen. Following a delay of $500 \mathrm{msec}$, the next stimulus appeared. The stimuli were chosen randomly with the restriction that each stimulus be different from the previous one. If the subject pressed two keys within a short interval (e.g., an error response followed immediately by a correction response) or pressed a key other than one of the four specified responses, the following instruction appeared on the screen: "Make sure your fingers are on the right keys. Press 'Spacebar' to continue." In addition, if an anticipatory response was made (less than $150 \mathrm{msec}$ after stimulus presentation), the trial was immediately aborted and excluded from the analysis. The subject was allowed up to approximately 2 min to rest between blocks.

\section{RESULTS AND DISCUSSION}

\section{Blood Alcohol Concentration}

The mean levels of alcohol in milligrams per $100 \mathrm{ml}$ of blood measured by the breathalyzer 20 and $75 \mathrm{~min}$ after completion of the drink in the no-alcohol session were both zero; in the alcohol session they were 92.6 ( $S D=$ $26.3)$ and $106.9(S D=18.3)$, respectively. (The legal limit for driving in England is $80 \mathrm{mg}$ per $100 \mathrm{ml}$ blood.) 
It should be noted that the first reading was taken when blood alcohol would have been rising and the second was taken when it was falling, so that the mean peak blood alcohol concentration would have been slightly higher than either of these readings (see Drew et al., 1959).

\section{Overall Means}

The data from 3 subjects had to be omitted from the analysis. One had previously participated in a very similar choice reaction time experiment, another was unable to remain reasonably alert during the alcohol session, and the third misunderstood the instructions and did not attempt to respond to each stimulus as quickly as possible. In addition, two blocks of trials were lost from the alcohol session for 1 subject, who abandoned the experiment feeling unwell.

The overall results are presented in Table 1 . Three-way analyses of variance were conducted on the mean response times, standard deviations, and medians, with session order ( 2 levels: no-alcohol-alcohol and alcohol-noalcohol) as the between-subjects factor, and alcohol ( 2 levels) and response accuracy ( 2 levels: correct and error) as within-subjects factors.

For the mean response times, there was no effect of session order $[F(1,35)=1.79, p>.1]$, but there were significant effects of alcohol $[F(1,35)=28.63, p<.01]$ and response accuracy $[F(1,35)=43.77, p<.01]$. The only significant interaction was between session order and alcohol $[F(1,35)=29.56, p<.01]$. This can be best understood by taking as an example the means for correct responses only for the no-alcohol-alcohol group (555$552 \mathrm{msec}$ ) and the alcohol-no-alcohol group (571$488 \mathrm{msec}$ ). Thus, there is an average increase in mean correct reaction time due to alcohol of $40 \mathrm{msec}$ and an average decrease from the first to the second session of $43 \mathrm{msec}$. The absence of an overall session order effect indicates that alcohol does not interact with practice, that is, there is no particular overall advantage in receiving alcohol in the first or second session.

For standard deviations, there was no effect of session order $(F<1)$, but there were significant effects of alcohol $[F(1,35)=10.21, p<.01]$ and response accuracy $[F(1,35)=5.26, p<.05]$. From Table 1 it can be seen that alcohol increased variability, while correct responses were more variable than errors. Again, there was a ses-

Table 1 Overall Means

\begin{tabular}{|c|c|c|}
\hline & No Alcohol & Alcohol \\
\hline \multicolumn{3}{|c|}{ Correct } \\
\hline $\begin{array}{l}\text { Mean RT (msec) } \\
S D \text { (msec) } \\
\text { Median RT (msec) }\end{array}$ & $\begin{array}{l}522 \\
128 \\
503\end{array}$ & $\begin{array}{l}562 \\
149 \\
540\end{array}$ \\
\hline \multicolumn{3}{|c|}{ Error } \\
\hline $\begin{array}{l}\text { Mean RT (msec) } \\
S D \text { (msec) } \\
\text { Median RT (msec) }\end{array}$ & $\begin{array}{l}487 \\
116 \\
469\end{array}$ & $\begin{array}{l}520 \\
136 \\
502\end{array}$ \\
\hline Error Rate (\%) & 4.14 & 4.97 \\
\hline
\end{tabular}

sion order $\times$ alcohol interaction $[F(1,35)=17.57, p<$ $.01]$. There was an overall decrease in standard deviation of $27 \mathrm{msec}$ from the first to the second session.

When median reaction times were analyzed, rather than means, an identical pattern of results was obtained, that is, significant effects of alcohol $[F(1,35)=40.28, p<$ $.01]$ and response accuracy $[F(1,35)=52.45, p<.01]$ with an interaction between sesson order and alcohol $[F(1,35)=32.94, p<.01]$. The overall decrease in median response time from the first to the second session was 32 msec.

The error rates were subjected to a two-way mixed analysis of variance with session order and alcohol as factors. There was no effect of session order $[F(1,35)=$ $1.18, p>.1]$ but there was a significant effect of alcohol $[F(1,35)=8.18, p<.01]$ and no interaction $(F<1)$.

To summarize, responses are slower, more variable (but see below), and less accurate with alcohol, and are faster and less variable in the second than in the first session.

There is normally a monotonic relationship between mean correct response time and variability of correct responses. It is therefore important to ask if the increase in standard deviation with alcohol is more than that which is usually associated with slower responses. This was investigated by performing linear regressions on mean correct response times against standard deviations separately for no alcohol and alcohol. First, the correlation coefficients were .814 and .836 , respectively, confirming that there is a strong tendency for standard deviation to increase linearly with mean reaction time. Second, the linear regression equations were very similar, with slopes of .483 and .449 and intercepts of -124.2 and $-112.8 \mathrm{msec}$ for no alcohol and alcohol, respectively. From this analysis it can be concluded that there is no evidence that alcohol increases variability any more than would be expected for an increase in mean response time.

\section{Changes in Performance Within a Session}

It is possible that the effect of alcohol is more severe at the end of a long session than at the beginning (see, e.g., Gustafson, 1986; Wilkinson \& Colquhoun, 1968). To investigate this, mean reaction times for correct responses only and the error rates were analyzed in two separate analyses of variance with session order as the between-subjects factor and alcohol and block (10 levels) as within-subjects factors ( 36 subjects). The overall means are presented in Figures 1 and 2.

For response times, there was no effect of session order $(F<1)$, but there were highly significant effects of alcohol $[F(1,34)=32.33, p<.01]$ and block $[F(9,306)$ $=5.42, p<.01]$. There were interactions between session order and alcohol $[F(1,34)=44.18, p<.01]$ and between session order, alcohol, and block $[F(9,306)=$ $4.63, p<.01]$.

The effect of alcohol and the session order $\times$ alcohol interaction have already been discussed. It can be seen from Figure 1 that, in general, response time decreases 


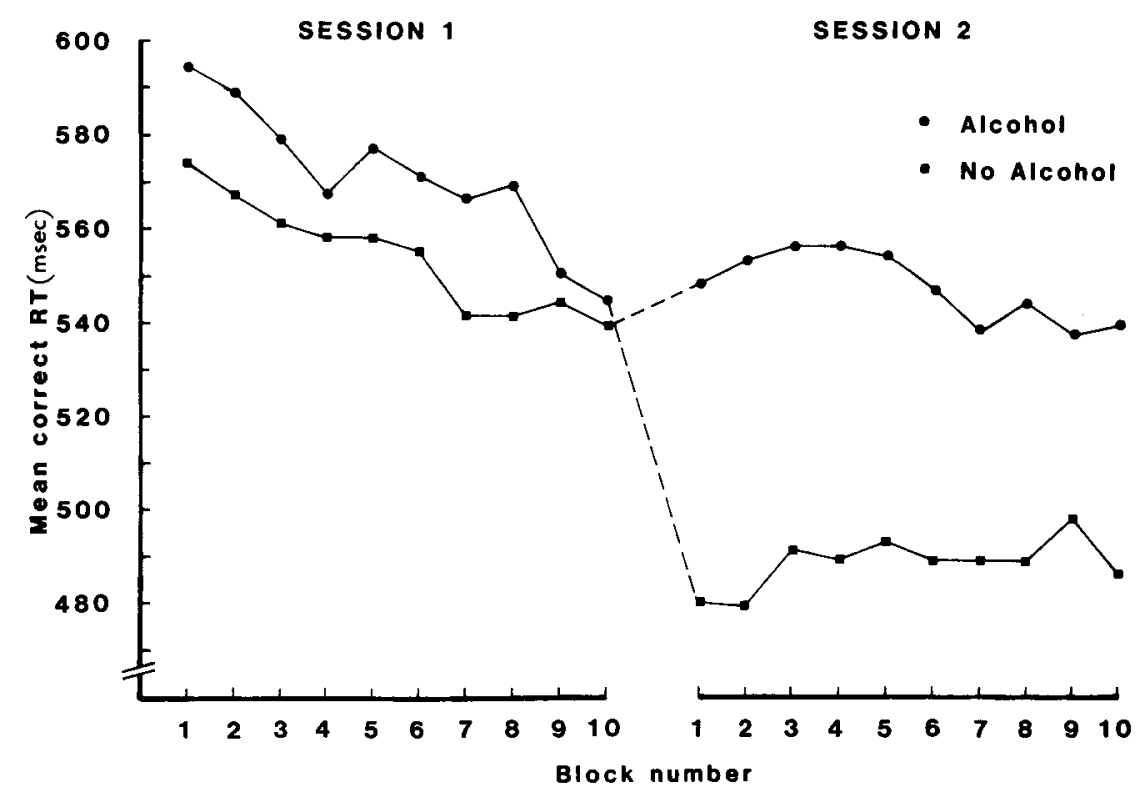

Figure 1. Mean correct response times as a function of block for Sessions 1 and 2, with and without alcohol.

over the session (the block effect). This is more marked on the first than on the second session, and, indeed, without alcohol on the second session, there is a slight increase leading to the three-way interaction. There is certainly no evidence from the mean correct response times to support the suggestion that the effect of alcohol is greater toward the end of a session, although it has to be remembered that blood alcohol concentration would not remain constant throughout an alcohol session (see Drew et al., 1959).

For errors, there were significant effects of alcohol $[F(1,34)=8.93, p<.01]$ and block $[F(9,306)=9.14$, $p<.01$, with no interactions. Figure 2 illustrates the general increase in error rate across each session, the ef- fect being similar with and without alcohol. This again indicates that the effect of alcohol is no more severe at the end than at the beginning of a 40-min session.

\section{Speed-Error Tradeoff Functions}

The above analysis does raise the question of SETOFs. Thus, the general change in performance from the 1st to the 10th block in a session (increased speed and errors) may be merely a movement along the SETOF. That is, subjects may operate in a different region of the SETOF without a change in actual performance efficiency implied by an alteration of the function itself. It is clear that this cannot be the case for the effect of alcohol as subjects are both slower and less accurate overall under alcohol.

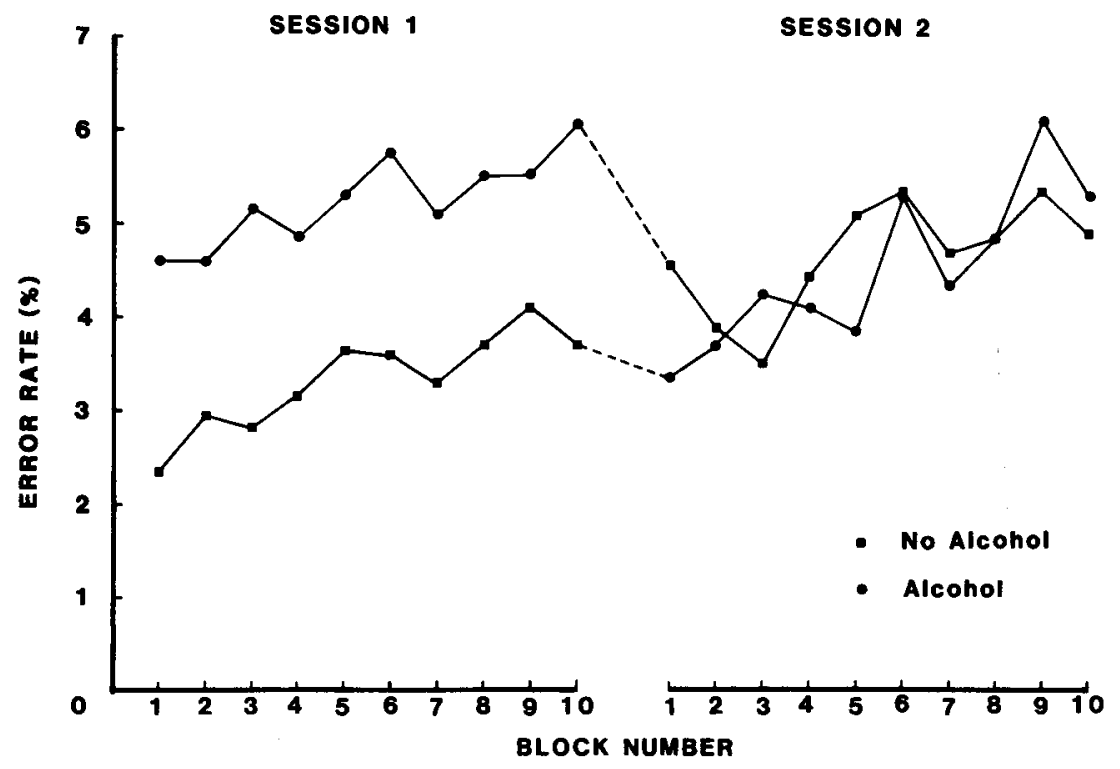

Figure 2. Error rates as a function of block for Sessions 1 and 2, with and without alcohol. 
(a)

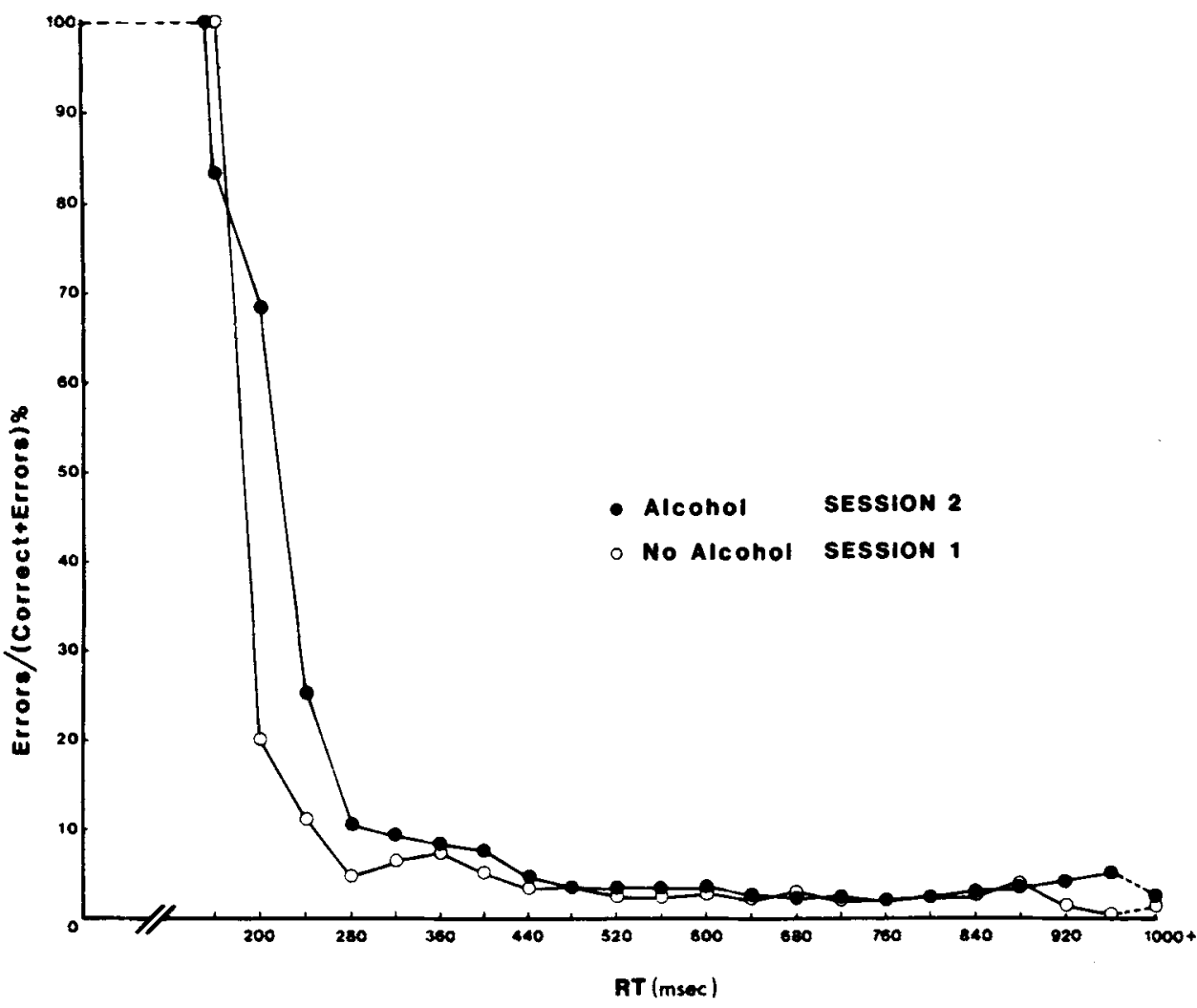

(b)

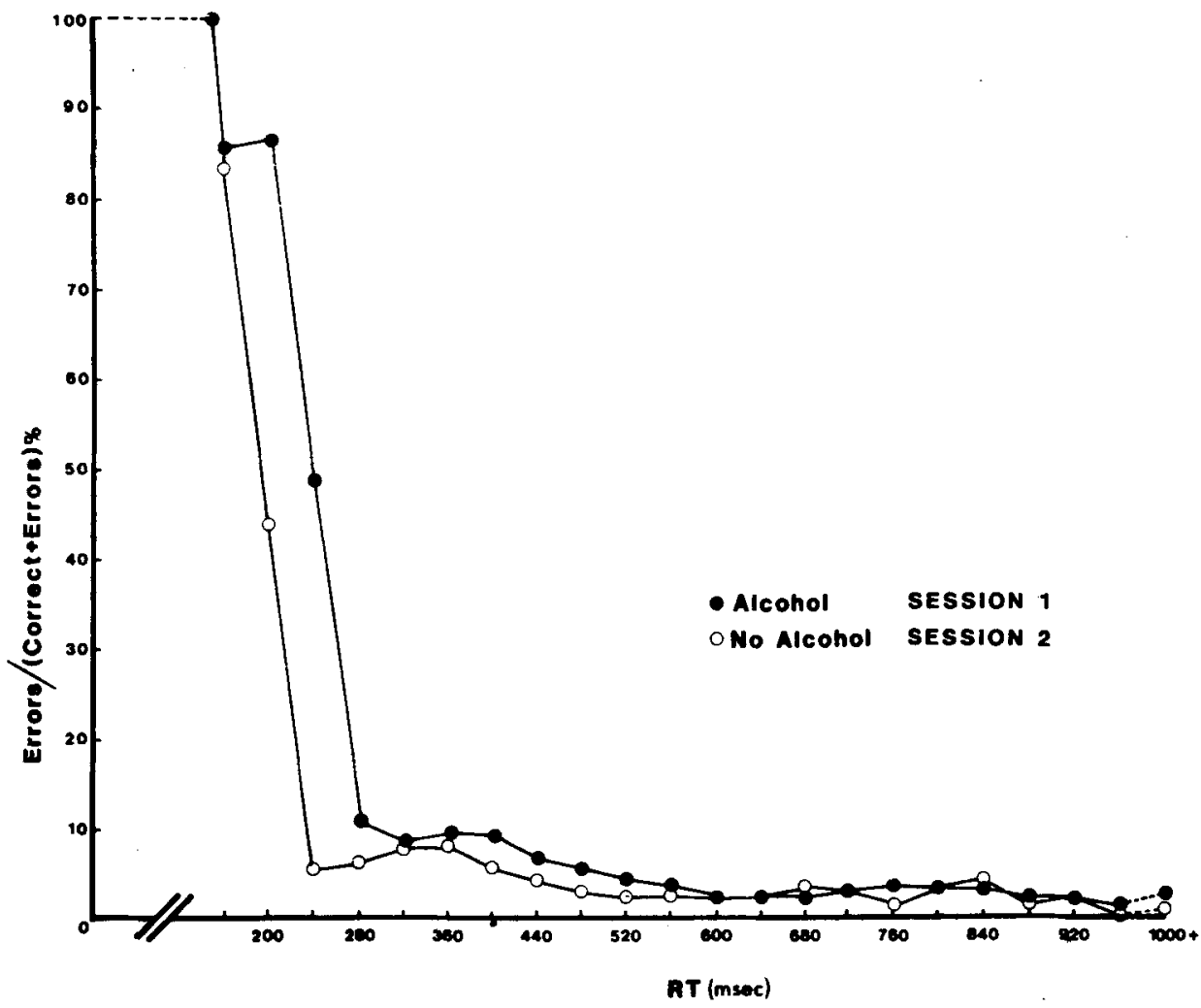

Figure 3. Speed-error tradeoff functions with and without alcohol. (a) No-alcohol-alcohol group, 19 subjects. (b) Alcohol-no-alcohol group, 18 subjects. 
The essential question here is how the SETOF is affected. To investigate this, functions were produced for each subject with and without alcohol by calculating the proportions of errors to total responses in reaction time bands of $40 \mathrm{msec}$. However, they were inconclusive, being far from smooth because of the very small number of errors and correct responses at the extreme ends of the function. The data were therefore combined across subjects to produce four SETOFs, that is, for the no-alcohol-alcohol order and alcohol-no-alcohol order, with and without alcohol. ${ }^{1}$

As can be seen from Figure 3, there was little effect of session order, that is, SETOFs do not change their position within the limits of practice given in this experiment (see also Rabbitt, in press). This is particularly striking when, for example, the mean correct reaction times for the four conditions are considered. (These were discussed earlier in relation to the session order $\times$ alcohol interactions.) For no alcohol and alcohol in Figure 3a, the means are 555 and 552 msec, respectively; in Figure $3 \mathrm{~b}$, the means are 488 and $571 \mathrm{msec}$, respectively. The SETOFs for the same condition (either no alcohol or alcohol) are thus very much more alike than would perhaps have been expected on the basis of the mean response times alone. Therefore, the data from all the subjects were combined to produce the overall SETOFs shown in Figure 4. First, it can be seen that the functions are very similar between 600 and $920 \mathrm{msec}$. For responses less than $600 \mathrm{msec}$, the SETOF for no alcohol is generally below that for alcohol. Thus, a response made within $600 \mathrm{msec}$ of stimulus presentation is more likely to be an error with alcohol than without. From Figure 4 it appears that, compared with no alcohol, the point of the lowest error rate for alcohol is further to the right along the reaction time axis. The overall reaction time distributions for correct and error responses are presented in Figures 5 and 6 , respectively. It should be remembered that the areas beneath the graphs are not equal (particularly for Figure 6) since alcohol increases the number of errors by $20 \%$ and decreases the number of correct responses by $0.9 \%$.

\section{Possible Models}

Within models of choice reaction time such as, for example, Fisher (1984), these effects of alcohol are most parsimoniously explained in terms of a decrease in the rate of accumulation of evidence (see Figure 7). This would show up as a shift in the intercept of the SETOF to the right along the reaction time axis. Both correct and error responses with alcohol take longer and are more variable than without alcohol. Responses made before the time required for a correct decision are more likely to be errors because they are based on insufficient evidence. If the ac-

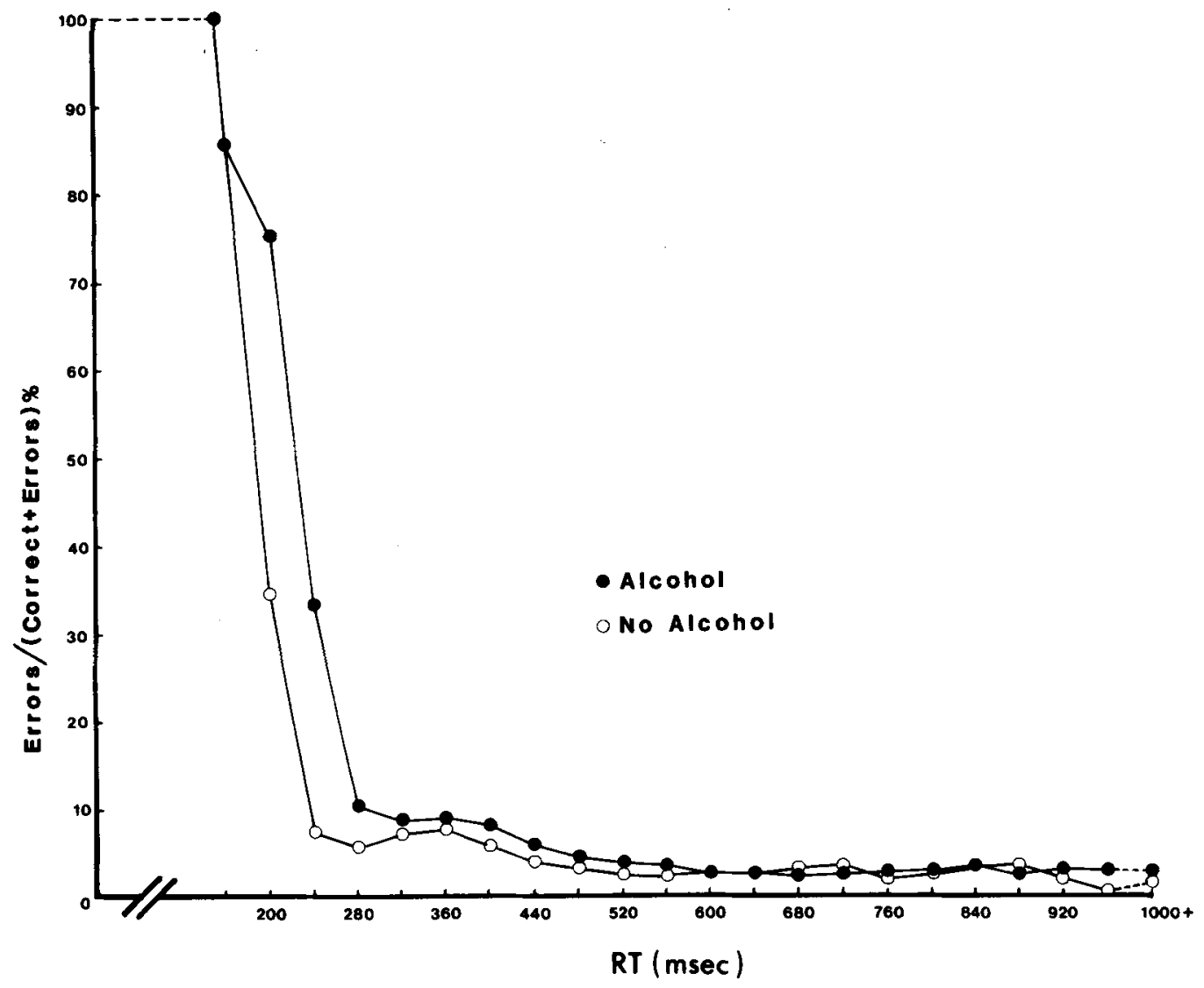

Figure 4. Speed-error tradeoff functions with and without alcohol (37 subjects). 


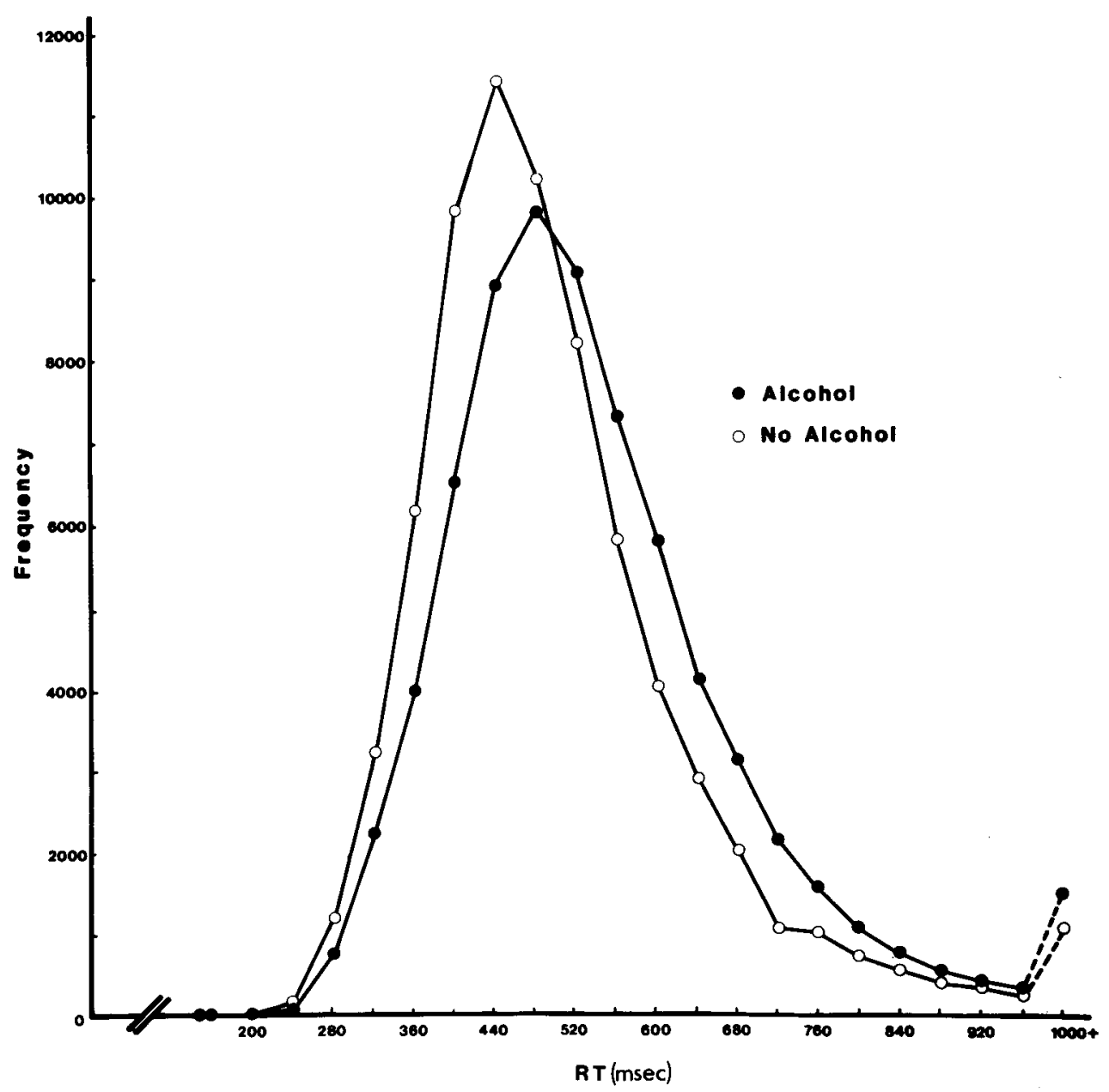

Figure 5. Reaction time distributions for correct responses.

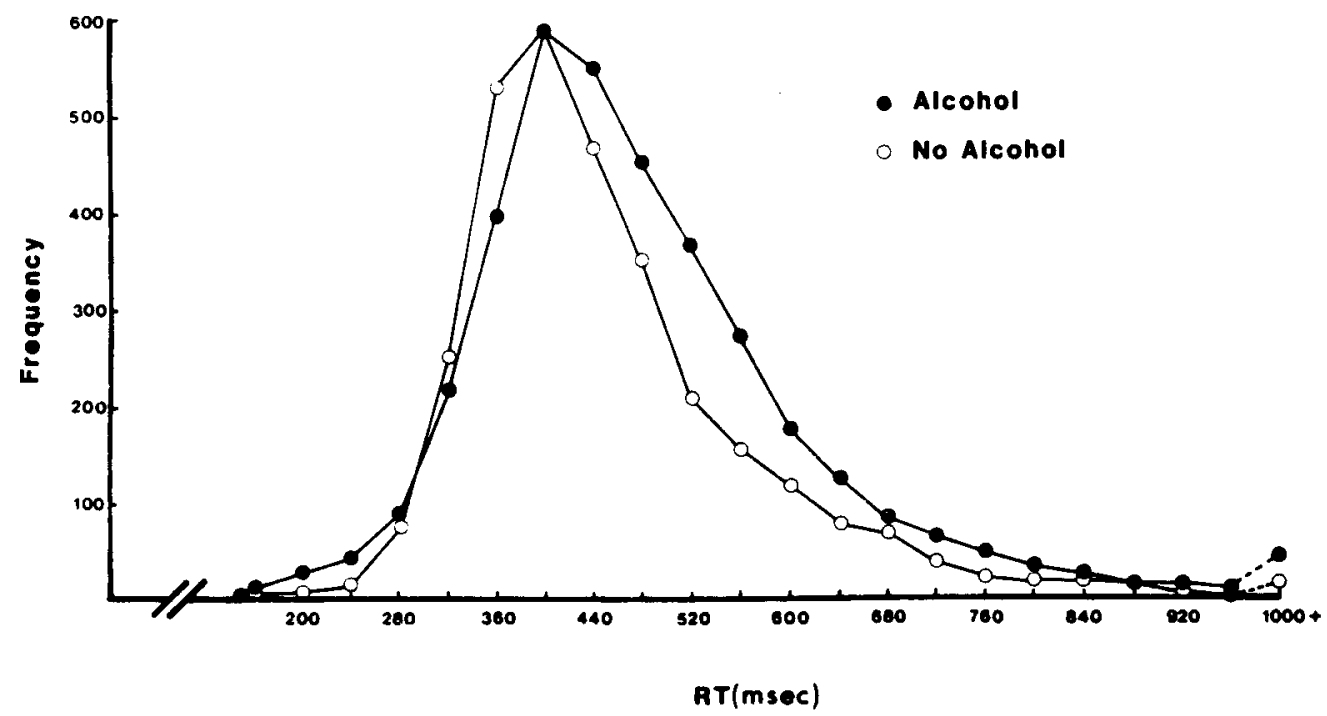

Figure 6. Reaction time distributions for error responses. 


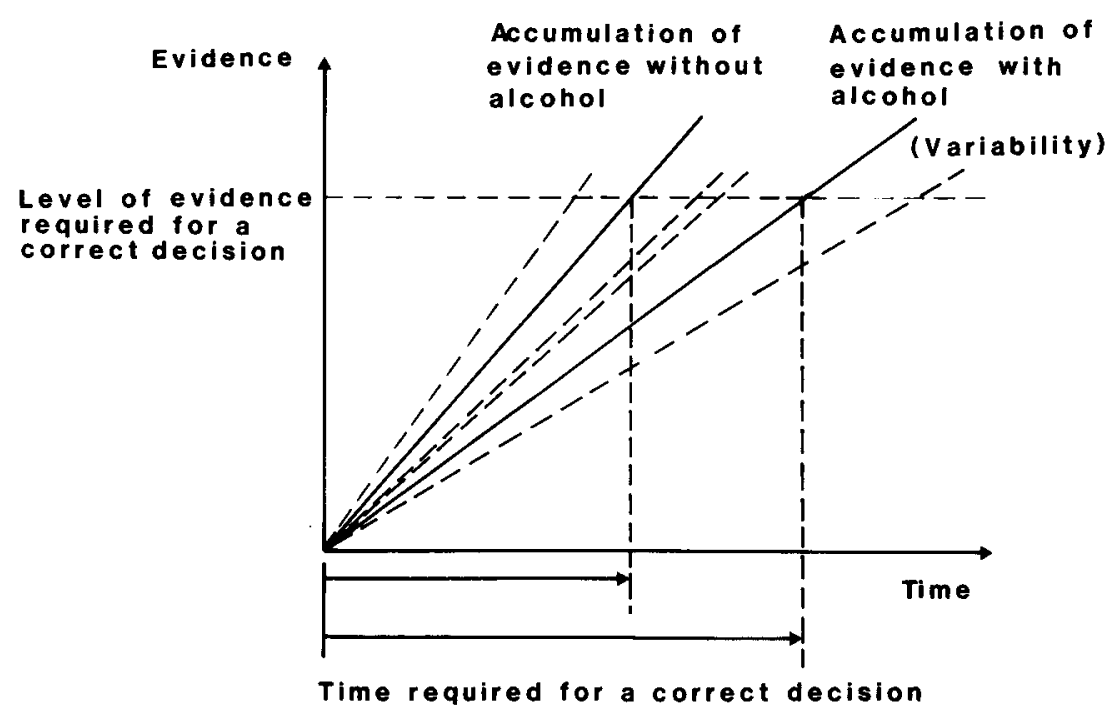

Figure 7. Model illustrating the possible effect of alcohol on speeded decision-making.

cumulation rate is lower with alcohol, it would follow that a response made within a certain time (which appears from Figure 4 to be approximately $600 \mathrm{msec}$ ) is more likely to be an error with alcohol than without alcohol.

Figure 7 is obviously an oversimplification and cannot, for example, account for the observation that reaction time distributions are positively skewed (see the mean-median differences of Table 1). Rabbitt (1979) suggested that the system "controls its maximum output rate to observe a speed-error trade-off, and controls its minimum output rate to avoid undesirably slow reaction bands"' (p. 136). Practice and age appear to affect the latter, that is, by altering the length of the "tail" of the reaction time distributions. This is consistent with the present experiment, in which there was no change in the overall shape of the SETOF between the two sessions (see Figure 3) but responses were slower and more variable in the first than in the second session.

\section{What Happens After an Error?}

It has already been suggested that errors are fast responses based on insufficient evidence. To respond as quickly as possible, subjects must make errors occasionally in order to ensure that they are operating very near their limits. Rabbitt (1979) has recently argued that in reaction time tasks, subjects "track" their SETOFs, that is, they gradually increase their speed until an error is made, then make an immediate adjustment to the amount of evidence required before responding, so that the response after an error is abnormally slow (see also Laming, in press).

The question of interest here is: How is this control mechanism affected by alcohol? For example, the increase in errors with alcohol could be due to a failure to make a large enough adjustment following an error so that although the next response is correct, as the subject increases speed again, the interval between errors decreases.
This was investigated by analyzing the errors and correct responses immediately following errors.

The mean response times were analyzed with session order (no-alcohol-alcohol; alcohol-no-alcohol) as the between-subjects factor and alcohol and trial type (5 levels: error responses [E], correct responses where the previous response was an error [EC], correct responses where the previous response was correct but the response before that was an error [ECC], and so on, up to correct responses occurring after an error followed by three correct responses [ECCCC]) as within-subjects factors. This revealed no effect of session order $[F(1,35)=1.61$, $p>.1]$ but significant effects of alcohol $[F(1,35)=$ $36.51, p<.01]$ and trial type $[F(4,140)=32.76, p<$ $.01]$. There were significant interactions between session order and alcohol $[F(1,35)=37.56, p<.01]$ and between alcohol and trial type $[F(4,140)=4.77, p<.05]$. Finally, the three-way interaction was significant $[F(4,140)=4.84, p<.05]$.

The means are presented in Table 2 , in addition to the remaining correct responses, included for comparison.

Table 2

Mean Response Times (in Milliseconds) for Errors and Responses Following Errors

\begin{tabular}{crcc}
\hline Session Order & \multicolumn{1}{c}{ Trial Type } & No Alcohol & Alcohol \\
\hline No Alcohol-Alcohol & E & 521 & 523 \\
& EC & 622 & 625 \\
& ECC & 576 & 565 \\
& ECCC & 567 & 564 \\
& ECCCC & 554 & 560 \\
Alcohol-No Alcohol & (Other Cs) & 551 & 547 \\
& E & 453 & 517 \\
& EC & 521 & 656 \\
& ECC & 490 & 584 \\
& ECCC & 482 & 575 \\
& ECCCC & 485 & 566 \\
& (Other Cs) & 486 & 565 \\
\hline
\end{tabular}


Subjects are slower with alcohol than without (the alcohol effect) and slower overall in the first than in the second session (reflected in the session order $\times$ alcohol interaction). The interaction between alcohol and trial type can be attributed to the greater effect of alcohol for EC trials than for the other types of trials $(+69 \mathrm{msec}[\mathrm{EC}]$ vs. $33 \mathrm{msec}$ [E], $42 \mathrm{msec}$ [ECC], $45 \mathrm{msec}$ [ECCC], $44 \mathrm{msec}$ [ECCCC]). (It should be noted that the interaction remained highly significant at $p<.01$ when correct responses only were analyzed.) The increase in reaction time following an error ( $E$ to $E C$ ) is $121 \mathrm{msec}$ with alcohol and $85 \mathrm{msec}$ without alcohol. Similarly, the three-way interaction is mainly the result of a difference in this increase from the first $(+120 \mathrm{msec})$ to the second $(+85 \mathrm{msec})$ session. It has already been suggested that practice does not greatly alter the rate of accumulation of evidence, so that the decrease in this difference from the first to the second session indicates that the adjustment made to the amount of evidence required following an error is initially large (when the subject is overcautious) but is reduced by practice. With alcohol, however, evidence accumulates at a slower rate, so that an identical adjustment to the response criterion following an error, with and without alcohol, can result in different effects $(+121$ and $+85 \mathrm{msec}$, respectively) on overall response time. $^{2}$

\section{Are Errors Distributed Randomly?}

Table 3 presents the mean numbers of each of the trial types in Table 2 (excluding the "Other Cs"). These were similarly analyzed with session order as the betweensubjects factor and alcohol and trial type as within-subjects factors. There was no effect of session order $[F(1,34)=$ $2.01, p>.1]$, but there were significant effects of alcohol $[F(1,34)=8.49, p<.01]$ and trial type $[F(4,136)$ $=42.46, p<.01]$. The only significant interaction was between alcohol and trial type $[F(4,136)=5.35$, $p<.01]$. The trial-type effect is, of course, merely the result of the loss of Trial Types 2-5 for every consecutive error and for every error on the last trial of a block, the loss of Trial Types 3-5 for every ECE sequence and for every error on the last or penultimate trial of a block, and so on. Thus, because of the overall increase in the number of errors with alcohol, an interaction between alcohol and trial type was expected. However, one interesting question is whether the observed numbers are different from those expected by chance given a particular error

Table 3

Mean Numbers of Errors and Responses Following Errors

\begin{tabular}{lrlc}
\hline \multicolumn{2}{l}{ Trial Type } & No Alcohol & Alcohol \\
\hline 1. & E & 80.0 & 97.6 \\
2. & EC & $76.6(75.4)$ & $90.4(91.2)$ \\
3. ECC & $72.3(71.7)$ & $84.3(84.0)$ \\
4. ECCC & 68.5 & 79.7 \\
5. ECCCC & 65.1 & 75.6 \\
\hline
\end{tabular}

Note-The figures in brackets are the mean numbers expected assuming a random distribution of errors. rate; that is, are the errors distributed randomly throughout the 2,000 trials? The number of consecutive errors was therefore investigated in the following way. For each subject, the expected number of Trial Type 2 was calculated, both with and without alcohol, by first assuming that, given an error, the probability of the next trial's being an error equals Number of errors/2000. Thus, the expected number of trials of Type 2 in a session equals Number of errors - (Number of errors $\times$ Number of errors $/ 2,000+10 \times$ Number of errors $/ 2,000$ ) (the latter part of the expression being the expected number of consecutive errors plus the expected number of errors at the end of a block).

An analysis of variance was conducted on these observed and expected numbers of trials of Type 2 with session order as the between-subjects factor and alcohol and observed versus expected as within-subjects factors. There were no effects of session order $[F(1,34)=2.05, p>.1]$ or of observed/expected $(F<1)$, but there was a significant effect of alcohol $[F(1,34)=8.56, p<.01]$. The only interaction was a highly significant one between alcohol and observed/expected $[F(1,34)=11.43, p<.01]$. From the means for Trial Type 2 in Table 3, it can be seen that this interaction is due to an increase in the observed number without alcohol, but a decrease with alcohol as compared with the expected numbers. This indicates that consecutive errors occurred less often than would be expected by chance without alcohol, but much more often than expected with alcohol. Because of the criterion shift implied by the increase in response time following an error (see Table 2), the result for no alcohol should not be surprising (see also Laming, in press). Thus, when an error is made and detected, the next response is unusually cautious as a result and therefore more likely to be correct than otherwise. With alcohol, however, consecutive errors occur more frequently than would be expected by chance, and there are at least two possible reasons for this. The first involves correction responses, which were observed by Rabbitt (1966) as occurring on average $500 \mathrm{msec}$ after error responses. In the present study, the subject was required to inhibit these and to make only one response to each stimulus. A correction response made more than $650 \mathrm{msec}$ after an error (that is, 500-msec response-stimulus interval + 150-msec "anticipation" period) would have been classified as another error, since each stimulus was different from the previous one. The increase in the number of consecutive errors with alcohol may therefore be attributed to a reduction in the ability to inhibit correction responses, or to an increase in their latency putting more of them in the time window allowed by the experimental procedure, or to a combination of both of these factors. The second possibility is that as processing is generally slowed down by alcohol (see, e.g., Figures 5 and 7), the time taken to detect an error and make the appropriate adjustment is increased, by which time a second error may have occurred.

These possibilities were investigated by dividing the consecutive errors (EEs) into those that were probably 
Table 4

Mean Numbers of Consecutive Errors

\begin{tabular}{lcc}
\hline & No Alcohol & Alcohol \\
\hline EE where R2 $=$ S1 & 1.31 & 3.31 \\
EE where R2 $<>$ S1 & 1.53 & 3.33 \\
\hline
\end{tabular}

due to correction responses (that is, when the second response was appropriate to the first stimulus) and those that could not be attributed to correction responses (that is, when the second response was inappropriate to the first stimulus)-see Table 4. An analysis of variance was conducted on these numbers of trials with session order as the between-subjects factor and alcohol and consecutive error type as within-subjects factors. There was no effect of session order $[F(1,34)=1.59, p>.1]$ or error type $(F<1)$, but there was a significant effect of alcohol $[F(1,34)=11.42, p<.01]$. There were no significant interactions.

First, the absence of an effect of error type is important as it indicates that correction responses were occurring more than $650 \mathrm{msec}$ after error responses (the ratio of error types being 1:1 rather than 1:3 that would be expected if correction responses did not occur). Second, the effect of alcohol (demonstrated by the present analysis, but more importantly in the previous analysis in which the overall increase in error rate with alcohol was taken into account) combined with the absence of any interactions suggests that alcohol increases the probability of both correction and noncorrection responses. As discussed earlier, both effects can be attributed entirely to slower processing with alcohol. Thus, the increase in the number of correction responses can be seen as the result of their increased latency due to alcohol, allowing more of them to be detected by the computer program (that is, those made over $650 \mathrm{msec}$ after an error). Similarly, for the second type of consecutive error (noncorrection responses), it is suggested that alcohol increases the time taken, first, to realize that an error has been made and, second, to take appropriate action by adjusting the response criterion.

An analysis was also carried out on the numbers of trials of Type 3, with session order, alcohol, and observed/expected as factors. ${ }^{3}$ There was no effect of session order $[F(1,34)=2.05, p>.1]$ or of observed/expected $[F(1,34)=1.54, p>.1]$, and no interactions. The only significant effect was that of alcohol $[F(1,34)=7.73, p<.01]$. From Table 3 it can be seen that for Trial Type 3 the observed values are slightly higher (although not significantly) than the expected values for both alcohol and no alcohol. Combined with the average difference of $16.5 \mathrm{msec}$ between the response times to Trial Types ECC and the other correct responses ("'Other Cs"), this indicates that the criterion adjustment following an error probably affects at least the following two responses (see Laming, in press). No further analyses were conducted on the numbers of trial types.

\section{CONCLUSIONS}

The overall results suggest an impairment in performance of a self-paced four-choice reaction time task after consumption of $1 \mathrm{ml}$ alcohol per kilogram of body weight, which cannot be attributed to a difference in response criterion only; responses are slower, more variable, and less accurate. There is an overall improvement in speed and variability, but no change in accuracy from the first to the second session, which could result from a reduction in the number of unnecessarily slow responses rather than from an improvement in processing rate. Analysis of the combined data from all the subjects confirmed that practice has little effect on the SETOF, whereas alcohol alters the function up to $600 \mathrm{msec}$, so that a response made within that time is more likely to be an error with alcohol than it is without alcohol.

Thus, conclusions similar to those of Jennings et al. (1976) have been reached using a different experimental paradigm and a different treatment of the data. The main results are not inconsistent with the view that alcohol decreases the rate of accumulation of evidence. In addition, the present results indicate that alcohol has some effect on the control over the upper rate of responding (see Rabbitt, 1979). Although an adjustment is made to the response criterion following an error both with and without alcohol, the increase in the number of the two types of consecutive error with alcohol suggests the following. First, error detection is slower with alcohol so that the appropriate adjustment is delayed, leading to an increase in the number of consecutive errors not attributable to correction responses. Second, alcohol may decrease the ability to inhibit a correction response to an error or may increase the latency of a correction response, both of which would lead to an increase in the number of consecutive errors attributable to correction responses. Further experimentation is required to distinguish between these last two possibilities.

\section{REFERENCES}

Brondbent, D. E. (1971). Decision and stress. London: Academic Press.

CARPENTER, J. A. (1962). Effects of alcohol on some psychological processes. Quarterly Journal of Studies on Alcohol, 23, 274-314.

Drew, G. C., Colquhoun, W. P., \& Long, H. A. (1959). Effects of small doses of alcohol on a skill resembling driving (MRC Memorandum No. 38). London: Her Majesty's Stationery Office.

Fisher, S. (1984). Stress and the perception of control. Hillsdale, NJ: Erlbaum.

GustAFson, R. (1986). Alcohol and vigilance performance: Effect of small doses of alcohol on simple visual reaction time. Perceptual \& Motor Skills, 62, 951-955.

HUNTLEY, M. S. (1972). Influences of alcohol and S-R uncertainty upon spatial localisation time. Psychopharmacologia (Berlin), 27, 131-140.

HunTley, M. S. (1973). Alcohol influences upon closed-course driving performance. Journal of Safery Research, 5, 149-164.

HuNTLEY, M. S. (1974). Effects of alcohol, uncertainty and novelty upon response selection. Psychopharmacologia (Berlin), 39, 259-266.

Jennings, J. R., WoOd, C. C., \& LAWRENCE, B. E. (1976). Effects 
of graded doses of alcohol on speed-accuracy tradeoff in choice reaction time. Perception \& Psychophysics, 19, 85-91.

LAMING, D. (in press). Some boundary conditions of choice-reaction performance. In A. F. Sanders (Ed.), Proceedings of Aachen Symposium on Choice Reaction Time and Stress. Hillsdale, NJ: Erlbaum.

LEONARD, J. A. (1959). Tactual choice reactions: I. Quarterly Journal of Experimental Psychology, 11, 76-83.

MAYLOR, E. A., \& RABBITT, P. M. A. (1987). Effect of alcohol on rate of forgetting. Psychopharmacology, 91, 230-235.

RABBITT, P. M. A. (1966). Errors and error correction in choice response tasks. Journal of Experimental Psychology, 71, 264-272.

RakBitr, P. M. A. (1979). Current paradigms and models in human information processing. In V. H. Hamilton \& D. M. Warburton (Eds.), Human stress and cognition: An information processing approach. New York: Wiley.

RABbitT, P. M. A. (in press). Models for effects of alcohol on skilled performance. In A. F. Sanders (Ed.), Proceedings of Aachen Symposium on Choice Reaction Time and Stress. Hillsdale, NJ: Erlbaum.

RABBITT, P. M. A., \& GowARD, L. (1986). Effects of age and raw IQ test score on mean correct and mean error reaction times in serial choice tasks. A reply to Smith and Brewer. British Journal of Psychology, 77, 69-73.

SChneIDer, W., DumaIs, S. T., \& ShIFFrin, R. M. (1984). Automatic and control processing and attention. In R. Parasuraman \& D. R. Davies (Eds.), Varieties of attention. New York: Academic Press.

Shiluto, M. L., King, L. E., \& Cameron, C. (1974). Effects of alcohol on choice reaction time. Quarterly Journal of Studies on Alcohol, 35, 1023-1034.

Tharp, Van K., JR., Rundell, O. H., Lester, B. K., \& Williams, H. L. (1974). Alcohol and information processing. Psychopharmacologia (Berlin), 40, 33-52.

Wilkinson, R. T., \& Colquhoun, W. P. (1968). Interaction of alcohol with incentive and with sleep deprivation. Journal of Experimental Psychology, 76, 623-629.

\section{NOTES}

1. Since responses made within 150 msec ("anticipations") were not included in the analysis, the first RT band (120-159 msec) was plotted as $150 \mathrm{msec}$, the second band $(160-199 \mathrm{msec})$ as $160 \mathrm{msec}$, the third band (200-239 msec) as $200 \mathrm{msec}$, and so on. Error rates of $100 \%$ in Figures 3 and 4 are perhaps surprising, since the task was four-choice (or indeed three-choice, given that the subject was told in advance that there were no immediate repetitions). Nevertheless, of the responses made between 150 and $159 \mathrm{msec}$, all were errors.

2. Again, we have to ask if this increase with alcohol is unexpectedly large, that is, more than a slowing of the rate of accumulation of evidence (see Figure 7) would lead us to expect. Following similar logic to that discussed above with regard to response variability, an appeal can be made to an analysis of individual differences. First, a linear regression was performed on the mean "other" correct response times against the mean increases in RT from E to EC without alcohol ( 37 subjects). The overall correlation was 0.666 , indicating that the $\mathrm{E}$ to $\mathrm{EC}$ reaction time difference is monotonically related to overall response speed or evidence accumulation rate. The slope of the regression equation was 0.727 and the intercept was $-293.1 \mathrm{msec}$. An analysis then conducted on the alcohol data revealed a slightly lower correlation coefficient of 0.539 , but a very similar slope $(0.773)$ and intercept $(-310.5)$. The conclusion must therefore be that it would be difficult to distinguish between the performance of 2 subjects with the same mean correct response time (a slow sober subject and a fast subject slowed by alcohol) on the basis of either the $\mathrm{E}$ to $\mathrm{EC}$ reaction time increase or (as established earlier) the variability of correct responses.

3. The expected numbers were calculated in the following way. First, the probability that the two trials after an error are "correct then error" equals $(2,000-$ Number of errors $) / 2,000 \times$ Number of errors/ 2,000 , so that the expected number of such sequences equals this probability multiplied by the number of errors, minus the expected number of errors on either the last or the penultimate trial of a block (that is, $20 \times$ Number of errors $/ 2,000$ ). Thus, the expected number of trials of Type 3 equals the observed number of trials of Type 2 minus the expected number of ECE sequences.

(Manuscript received January 30, 1987; revision accepted for publication May 7, 1987.) 